\title{
Potential Applications of Microfluidics to Acute Kidney Injury Associated with Viral Infection
}

\author{
Holly Ryan (i) ${ }^{1,2}$ and Chelsey S. Simmons (iD) ${ }^{1,2,3}$ \\ ${ }^{1}$ J. Crayton Pruitt Family Department of Biomedical Engineering, Herbert Wertheim College of Engineering, University of \\ Florida, PO Box 116250, Gainesville, FL 32611, USA; ${ }^{2}$ Department of Medicine, College of Medicine, University of Florida, \\ Gainesville, USA; and ${ }^{3}$ Department of Mechanical and Aerospace Engineering, Herbert Wertheim College of Engineering, \\ University of Florida, Gainesville, USA
}

(Received 4 June 2020; accepted 19 August 2020; published online 31 August 2020)

Associate Editor Anubhav Tripathi oversaw the review of this article.

\begin{abstract}
The kidneys are susceptible to adverse effects from many diseases, including several that are not tissue-specific. Acute kidney injury is a common complication of systemic diseases such as diabetes, lupus, and certain infections including the novel coronavirus (SARS-CoV-2). Microfluidic devices are an attractive option for disease modeling, offering the opportunity to utilize human cells, control experimental and environmental conditions, and combine with other onchip devices. For researchers with expertise in microfluidics, this brief perspective highlights potential applications of such devices to studying SARS-CoV-2-induced kidney injury.
\end{abstract}

Keywords-Severe acute respiratory syndrome, bioMEMS, Kidney failure, Organ-on-a-chip.

\section{INTRODUCTION}

Acute kidney injury (AKI) has been described as a common complication of the novel severe acute respiratory syndrome coronavirus 2 (SARS-CoV-2) infection. In one chart review from South Korea, AKI was found to be a complication in $9.2 \%$ of all patients with confirmed infection and in $61.5 \%$ of those admitted to intensive care. ${ }^{18}$ Various mechanisms have been proposed to explain how SARS-CoV-2 may cause AKI, from off-target effects of the inflammatory response to direct infection and death of kidney cells, though no clear correlation between infection and progression to AKI has been established. ${ }^{53}$ Further research is nec-

Address correspondence to Chelsey S. Simmons, J. Crayton Pruitt Family Department of Biomedical Engineering, Herbert Wertheim College of Engineering, University of Florida, PO Box 116250, Gainesville, FL 32611, USA. Electronic mail: css@ ufl.edu essary to investigate the effects of SARS-CoV-2 on the kidney and to assist in the development of treatments to avoid AKI.

Microfluidic $(\mu \mathrm{F})$ and "kidney-on-a-chip" systems are particularly well-suited to investigate AKI associated with the novel SARS-CoV-2 virus. In addition to isolation of complex biological variables for mechanistic investigations, such kidney-mimicking $\mu \mathrm{F}$ platforms can utilize human cells at scale for the rapid and high-throughput analysis demanded by this global crisis while recapitulating the dynamic microenvironment of the kidneys. This brief perspective highlights pathophysiological features of SARS-CoV-2 and its resulting disease, COVID-19, that are relevant to studies in kidney-mimicking $\mu \mathrm{F}$ platforms and suggests applications of such devices to investigations of SARSCoV-2-induced AKI.

\section{MIMICKING KIDNEY TUBULES FOR APPLICATIONS TO SARS-COV-2}

\section{Review of Kidney Anatomy and Physiology}

The kidney's basic blood-filtering unit is the nephron, composed of a long tubule through which the aqueous "filtrate" is separated from the blood flow. The tubule is lined with several types of renal cells that are responsible for transporting additional wastes into the filtrate and for recovering small proteins, sugars, and other substances back into the blood. The apical surfaces of these cells are exposed to fluid shear forces of about 1 dyne $/ \mathrm{cm}^{2}$ from filtrate flowing through the lumen $;{ }^{37}$ meanwhile, the basal surfaces are in contact with a stationary basement membrane composed of extracellular matrix (Fig. 1a). The apical and basal 
(a)

(b)
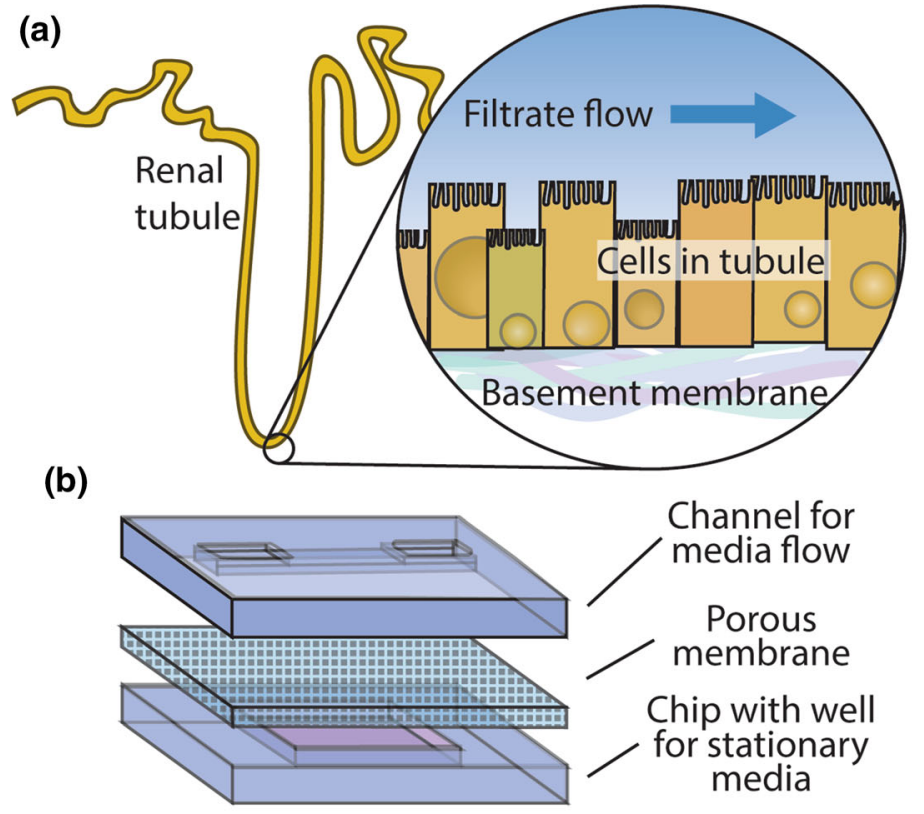

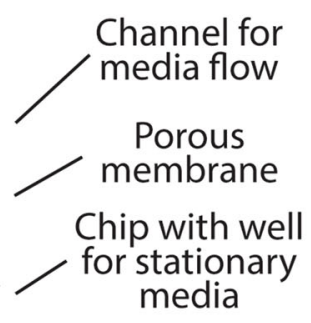

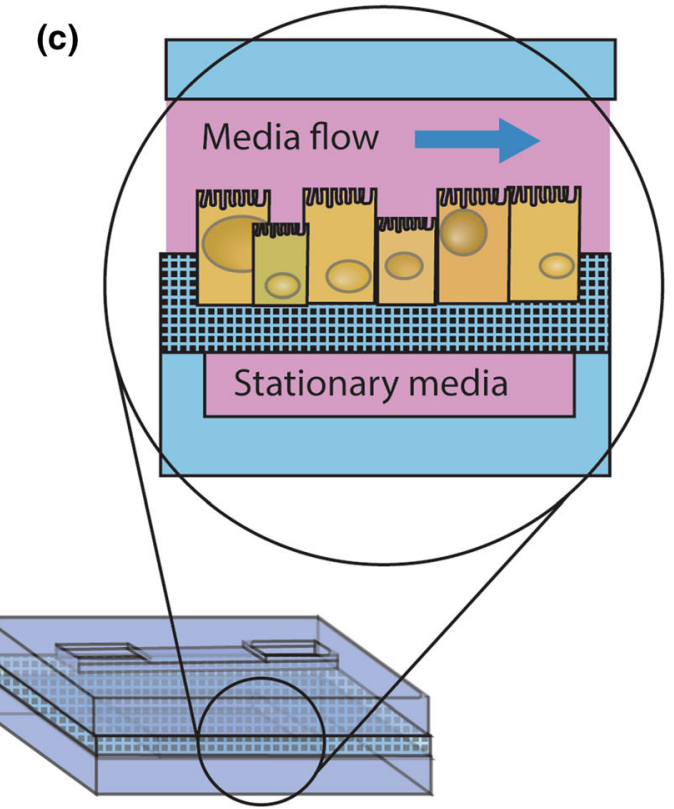

FIGURE 1. (a) Schematic of tubule epithelial cells. On their apical (top) surfaces, the cells comprising the renal tubules are exposed to shear stress from the constant laminar flow of the filtrate, while their basal (bottom) surfaces are anchored to the stationary basement membrane. The fluid shear stress from the filtrate in the lumen is an important signal directing the cells' polarity. When tubule cells are cultured in conditions where this stress is absent, they fail to develop into their characteristic elongated shape, and their cell surface markers are distributed evenly around the plasma membranes instead of localizing to a specific region. $(b-c)$ Schematic of standard microfluidic device that supports epithelial cell polarization. Cells are plated in an upper channel through which fluid can flow, separated from a stationary well by a porous membrane. The contents of the stationary well simulate the interstitial space, while cells are seeded onto the membrane. Images not to scale.

surfaces of tubule cells are highly differentiated when coaxed to "polarize"; for example, microvilli are present on the apical surface, but not on the basal surface. Additionally, some cell surface channels and enzymes are restricted to a particular region of the cell membrane, such as $\mathrm{Na}^{+}-\mathrm{K}^{+}$-ATPase, which is only present at the apical surface. ${ }^{52}$

Kidney tubule cells also experience fluid flow and concomitant shear stress. Multiple $\mu \mathrm{F}$ platforms have shown that the presence of shear stress is not only a necessary stimulus for cells to maintain polarity, ${ }^{20,22}$ but it also contributes to cell viability ${ }^{21}$ and cytoskeletal organization. $^{20,21}$ Additionally, exposure to shear stress improves the ability of cell monolayers to maintain tight junctions, leading to higher barrier integrity than is observed in cells from static culture. ${ }^{25}$ Exposure to shear stress has also been shown to improve cells' ability to recover from drug administration, ${ }^{22,25}$ suggesting a behavioral as well as a phenotypic response to shear stress. In devices modeling renal tubules, the fluid shear stress is generally set to be between 0.2 and $5 \mathrm{dyne} / \mathrm{cm}^{2}$ to model the rate of filtrate flow, which is achieved with channels typically $\sim 1 \mathrm{~mm}$ wide and $100 \mu \mathrm{m}$ deep. ${ }^{20-22,25}$ Since pressure gradients across kidney tubules are small, ranging from $\sim 13 \mathrm{mmHg}$ in the proximal tubules to $\sim 7 \mathrm{mmHg}$ in the collecting ducts in rats ${ }^{48}$ pressure gradients across devices are typically designed to be small as well.

\section{Device Design}

Kidney "spheroids", spontaneously organized cells in 3D that mimic tubule polarization and organization, have been in wide use since the 1980s but are not amenable to shear stress conditions within the spheroid interior. To ensure shear flow conditions in the tubule "lumen" and maintain cells in a monolayer for simple visualization, the most common design for a kidneymimicking $\mu \mathrm{F}$ device is a sandwich of two chips of a non-porous material with a porous membrane between them $^{20-22,25,52}$ (Figs. 1b and 1c). In lieu of synthetic porous membranes, protein-derived hydrogels made of collagen or basement membrane extract ${ }^{28,29,35,49,51}$ allow for the study of extracellular matrix remodeling and 3D migration, both of which occur during fibrosis. Once the cells have attached, media can be pumped through the channel at a rate and pressure similar to that present in the renal tubules. The materials used to construct the device, as well as the dimensions of the different components, vary depending on study design. In addition to the multitude of devices developed in research labs, commercial platforms are available 
including the OrganoPlate ${ }^{35,44,49,50}$ (Mimetas, Netherlands) and the Kidney-Chip ${ }^{22}$ (Emulate, US).

Advanced 3D printing techniques now afford more options for culturing spheroids within printed hydrogel structures $^{26}$ and for crafting sophisticated kidneymimicking geometries, ${ }^{17,42}$ but $\mu \mathrm{F}$ remain a powerful tool for rapid, high-content investigations of AKI associated with SARS-CoV-2.

\section{Cell Source}

There are many different cell types present in the kidneys and many sources of cells for in vitro models, and the choice of which to use will depend on the biological hypothesis at hand. Immortalized kidney cell lines, such as Madin-Darby Canine Kidney (MDCK) cells, are often used for proof-of-concept studies to design and test kidney-on-a-chip systems $\mathrm{s}^{37,43,44,50}$ because they are readily available from commercial suppliers and can generally be passaged many times. However, cells from human sources are likely to afford more relevant gene expression for studies of direct SARS-CoV-2 infection and of the progression of COVID-19 than cells from animal sources would be. Coronaviruses enter cells via fusion with the angiotensin-converting enzyme 2 (ACE2) receptor; $3,15,30,33,45$ therefore, differences in expression of this receptor between species would affect a model's suitability for simulating human disease. Canines, the source of MDCK cells, can be infected by some coronaviruses but appear to have low susceptibility to SARS-CoV-2. ${ }^{40}$ Mice also have low susceptibility to coronaviruses, which is well documented from earlier experiments with SARS-CoV, the strain of coronavirus responsible for the 2002-2004 SARS outbreak. This virus was highly pathogenic in humans but was not able to induce severe disease in mice in the absence of genetic modifications. ${ }^{11}$

For COVID-19 investigations, human cells are likely to be the most relevant. Human renal proximal tubule epithelial cells (RPTECs) are commonly used in renal $\mu \mathrm{F}$ studies because they are commercially available isolated from normal donor kidneys, and they contain important transporters for reuptake of glucose and other molecules from the filtrate back into the blood. ${ }^{12}$ ACE2-related genes are highly expressed in proximal straight tubule cells, ${ }^{34}$ so cultures of these cells from human donors may be especially suited to SARS-CoV-2 studies. Another advantage to RPTECs is that they are highly sensitive to toxic effects from drugs, making them desirable for studies of drug metabolism as discussed in "Drug Screening and Toxicity" section. Last, RPTECs have been immortalized via telomerase reverse-transcriptase modification, and this commercially available line (RPTEC-TERT-1) retains similar responses to toxic stimuli with improved doubling capacity compared to primary cells. ${ }^{41}$ Table 1 lists some of the most common sources of human RPTECs, along with example $\mu \mathrm{F}$ applications.

While RPTECs are likely to be useful for fundamental investigations, patient-specific cells could prove useful to understand the large disparities between asymptomatic SARS-CoV-2 infections and development of severe COVID-19. Human induced pluripotent stem cells (iPSCs) can be differentiated into parenchymal cells while conserving genes that often confer disease-like in vitro phenotypes. iPSCs have been widely differentiated into kidney-lineage cells, i.e. nephron progenitor cells (NPCs), and are increasingly used in the formation of spontaneously assembling kidney organoids. ${ }^{14}$ iPSC-derived NPCs could be useful for understanding patient-specific pathophysiology and for screening targeted therapies, given the dramatic variability in disease presentation among COVID-19 patients.

Additional renal cell types, including podocytes and fibroblasts, are often incorporated into kidney-mimicking devices. Podocytes are cells that interface with the glomerulus, where filtrate is first removed from the blood, and they also highly express ACE2 receptors. ${ }^{34}$ Injury to the podocytes causes the barrier between the blood and the filtrate to leak, allowing proteins such as albumin to leave the blood and be lost in the urine. Podocytes are often used in studies of kidney injury, ${ }^{35,36}$ and spheroids of RPTECs and podocytes are even available commercially (Tempo Bioscience). Fibroblasts are present in most tissues including the kidneys and are responsible for maintaining extracellular matrix. They are typically used in kidney-on-achip devices either as a source of growth factors ${ }^{24}$ or in studies of fibrosis, when fibroblasts are uncontrollably activated and form excessive scar tissue in an organ. ${ }^{31}$ Many studies also use endothelial cells to investigate the interaction between renal cells and the blood vessels associated with them throughout the kidney, since fibrogenic substances can induce endothelial-mesenchymal transition. ${ }^{31}$ Fibrosis is discussed further in "Fibrosis" section.

\section{Utilizing $\mu F$ Kidneys-On-a-Chip}

The primary metrics of "successful" renal tubule recapitulation in vitro are typically cell polarization and barrier integrity. Cell polarization includes localization of tight junction proteins to the apical side, which are often visualized by fluorescence microscopy by fixing and immunostaining cells directly on the porous membrane of the device. ${ }^{22,52}$ Barrier integrity, which may be reduced in the presence of pathogens or drug metabolites, is often analyzed by perfusing the 
TABLE 1. Common sources of human renal proximal tubule epithelial cells used in different applications of kidney-on-a-chip devices.

\begin{tabular}{|c|c|c|}
\hline Application & Cell source & References \\
\hline 3D model for future small-molecule studies or implantation & Immortalized (HK2) & 42 \\
\hline Artificial filtration device & Primary (from Lonza) & 13 \\
\hline \multirow[t]{2}{*}{ Effects of mechanical stimuli and flow } & Primary (from Biopredic) & 22 \\
\hline & Conditionally immortalized (ciPTEC-OAT1) & 50 \\
\hline \multirow[t]{3}{*}{ Permeability and absorption } & Immortalized (HK2) and primary (from Lonza) & 12 \\
\hline & Primary (from Sigma) & 49 \\
\hline & Conditionally immortalized & 23 \\
\hline \multirow[t]{2}{*}{ Small-molecule induced kidney injury and fibrosis } & Conditionally immortalized (ciPTEC-OAT1) & 44 \\
\hline & Immortalized (HK2) & 58 \\
\hline \multirow[t]{3}{*}{ Metabolites } & Immortalized (RPTEC-TERT-1) & 32 \\
\hline & Primary (from ATCC) & 46 \\
\hline & Primary (in-house from discarded tissue) & 4 \\
\hline
\end{tabular}

HK-2 human kidney 2, ciPTEC-OAT1 conditionally immortalized proximal tubule epithelial cells expressing organic anion transporters 1 , RPTEC-TERT-1 renal proximal tubule epithelial cells immortalized using human telomerase reverse transcriptase.

channel of the device with a fluorescent tracer and measuring leakage from the flow chamber into the static chamber over time..$^{28,29,35,42,49,51}$ This technique is also used in devices that use hydrogels to simulate the interstitial space. Immunostaining and fluorescence microscopy of cell-cell junction proteins like claudin, occludin, and E-cadherin can also be used as a proxy for barrier integrity. ${ }^{8}$

Loss of polarity and decrease in barrier integrity are signs of epithelial cell dysfunction and serve as useful assays during kidney-on-a-chip experiments. These and other changes can be visualized via microscopy, including changes in cellular morphology such as the development of visible "virus factories" within cells upon infection. ${ }^{52}$ For additional analyses, $\mu \mathrm{F}$ devices can be disassembled and/or cell lysate collected for cellular and molecular assays. For example, in the setting of viral infection, cells generally alter their expression of cytoskeletal proteins, ${ }^{59}$ which can be assessed by changes in gene-expression, protein-expression, and/or protein localization. Kidney-mimicking $\mu \mathrm{F}$ devices are compatible with a wide range of cellular, molecular, and functional assays to investigate biological hypotheses of interest related to AKI.

\section{ACUTE KIDNEY INJURY APPLICATIONS ASSOCIATED WITH SARS-COV-2}

While it remains debated, there are several conjectures as to how SARS-CoV-2 might induce AKI. Here, we examine several proposed mechanisms and examples of microfluidic devices that have been successfully employed to study similar pathologies. Since microfluidic devices are widely used to investigate inflammatory, fibrotic, and hematological diseases in addition to renal function, they could be of great value in identifying the mechanisms behind SARS-CoV-2induced kidney injury and in screening potential therapeutics. In addition, while many organs in addition to the kidneys are involved in systemic regulation of blood pressure and fluid/electrolyte balance that may impact kidney function, standard mouse models that may otherwise be used for systems-level pharmacology investigations may not be relevant to COVID-19 because of pertinent differences in ACE2 receptor and other related genes. Inbred, genetically modified mouse models are unlikely to represent patient diversity, creating a niche for kidney-mimicking $\mu \mathrm{F}$, potentially in combination with other organs-on-a-chip, to assist with preclinical screening of novel therapeutics for COVID-19.

\section{Direct Viral Infection}

The ability of SARS-CoV-2 to target lung tissue has been attributed to this tissue's relatively high expression of the ACE2 receptor, which the virus uses to enter the cells. ACE2 is also abundant in renal cells, 9,34 and renal cells were observed to take up viral particles in the setting of viral infection associated with the coronavirus strain that caused the SARS epidemic of 2002-2004. ${ }^{33}$ To mitigate AKI associated with SARSCoV-2, microfluidics could be used to investigate if and how renal cells respond to infection, leveraging previous applications to other viruses. In a study by Wang et al., pseudorabies viruses were shown to cause loss of polarity and to disrupt tight junctions between renal cells; importantly, cells cultured in the microfluidic platforms took longer to display evidence of viral pathology than did cells cultured in static platforms. ${ }^{52}$ $\mu \mathrm{F}$ platforms are thus well-suited to investigate microenvironmental conditions, cell types, and genotypes that lead to more severe SARS-CoV-2 infection 
and to test hypothesized antiviral treatments. However, some researchers with experience in microfluidics may not be familiar with virology research and the safety standards necessary to work with hazardous pathogens. We direct such readers to guidelines on biosafety level 3 laboratory design ${ }^{5}$ and to a recent report from the Centers for Disease Control and Prevention on biosafety precautions for handling SARS-CoV-2, specifically. ${ }^{19}$

\section{Drug Screening and Toxicity}

Considerable use has been made of microfluidic devices in the field of drug discovery, in both direct studies of therapeutics on the kidneys and in consideration of nephrotoxic side effects. SARS-CoV-2 infection involves ACE2 receptors, which are a known component of the renin-angiotensin system (RAS) that regulates blood pressure and fluid/electrolyte balance with extensive involvement of the kidneys. Proposed therapeutics may modulate components of the RAS in an effort to control SARS-CoV-2 infection, which would necessitate screening for potential effects on renal function. Conversely, patients may already be taking drugs like ACE-inhibitors for treatment of high blood pressure, and the effects of these drugs in modulating direct SARS-CoV-2 infection are of interest. In addition, proposed antivirals and their metabolites may inadvertently impair kidney function, as with the infamous antiviral acyclovir. ${ }^{1}$ Drug discovery studies using kidney-mimicking $\mu \mathrm{F}$ have examined the effects of drugs on monolayer formation ${ }^{23}$ and the effects of drug metabolism by one organ on the function of another, $4,32,46,47$ and similar strategies could be used for preclinical investigation of COVID-related therapies. Drug metabolism studies are also often accomplished by linking several types of microfluidic devices to represent different organ systems, ${ }^{7,27}$ for example linking a kidney-on-a-chip to a liver-on-a-chip to simulate metabolites being carried to the kidney.

\section{Inflammatory Cascades and Blood Clotting}

Inflammation is a key symptom of SARS-CoV-2 infection, often leading to pulmonary edema and death. ${ }^{10,30,54,55}$ Off-target effects of the inflammatory response, including cytokine storm, formation of microthrombi, and immune complex deposition have been proposed as possible contributors to the development of AKI. ${ }^{15}$ Similar to linking kidney-mimicking devices to liver-mimicking devices to examine the renal effects of metabolites, kidney-mimicking chips could be linked via direct proximity or via supernatant to activated immune cells, which are often used in microfluidic studies of chemotaxis ${ }^{38}$ or cytokine pro- filing. ${ }^{39}$ Similarly, clotting studies of microthrombi associated with COVID-19 are possible in a microfluidic platform. In microvascular studies, devices have been coated with a thrombogenic material such as tissue factor and then perfused with whole blood, triggering platelet activation and coagulation. ${ }^{2}$ To study the effects of platelet activation on renal cells, media containing normal platelets could be exposed to a thrombogenic stimulus, transferred to a kidney-on-achip device, and observed for the effect of activated platelets on renal cells.

\section{Fibrosis}

Pulmonary fibrosis, or deposition of excess extracellular matrix in the lungs, is the result of excessive inflammation and one of the most commonly observed symptoms in SARS-CoV-2 infection. ${ }^{54,56,57}$ Since the virus likely displays a tropism for the kidneys, there may be a risk for renal fibrosis in the setting of infection, as well. Though understanding is still growing, observed histopathological features of kidneys from COVID-19 patients to date include edema, ${ }^{30,55}$ shedding of tubular cells, ${ }^{55}$ and accumulation of protein in tubule lumens. ${ }^{55}$ Given the propensity of tubular edema and protein accumulation to progress to renal fibrosis, it seems likely that renal fibrosis could be linked to SARS-CoV-2-induced AKI, potentially involving epithelial-to-mesenchymal transition (EMT) and contributions to fibrosis from tubule cells themselves. ${ }^{58}$ Other reviews have discussed microfluidic devices for fibrosis modeling in general ${ }^{6}$ or for specific organs, ${ }^{16}$ and such platforms could be useful to simulate and investigate renal fibrosis associated with COVID-19. Combinatorial investigations of mechanistic interactions between parenchymal cells, fibroblasts, and individual soluble factors could be done efficiently in $\mu \mathrm{F}$ platforms to gain a better understanding of these complex interactions in AKI.

\section{SUMMARY AND OUTLOOK}

Microfluidics provide a flexible platform for studying renal cells that replicate in vivo-like shear stresses while providing potentially high-content and patientspecific data to inform the fast-moving fields of cell biology and drug development, for the current pandemic and in the future. Many engineering and biomedical labs are already experts in studying epithelial cells in kidney-mimicking conditions, and this expertise could readily be applied to the pandemic by investigating AKI associated with COVID-19. In addition, researchers experienced in in vitro platforms for virology research could leverage ready-made or- 
gan-on-a-chip products from commercial suppliers such as Mimetas ${ }^{35,44,49,50}$ or Emulate ${ }^{22}$ to replicate fluid shear stresses in their relevant investigations. New advances in SARS-CoV-2 research could be made quickly and efficiently with a more widespread use of microfluidics technology, leading not only to a better understanding of this disease and its impacts on kidney cells, but also to the development of better treatment options.

\section{ACKNOWLEDGMENTS}

This work has been partially supported by National Institutes of Health T32DK104721. We thank Dr. Z. Hugh Fan for providing valuable discussion and insights.

\section{CONFLICT OF INTEREST}

Holly Ryan and Chelsey S. Simmons declare that they have no conflicts of interest.

\section{DECLARATIONS}

No human nor animal studies were carried out by the authors for this article.

\section{REFERENCES}

${ }^{1}$ Becker, B. N., et al. Rapidly progressive acute renal failure due to acyclovir: case report and review of the literature. Am. J. Kidney Dis. 22:611-615, 1993.

${ }^{2}$ Brouns, S. L. N., I. Provenzale, J. P. Van Geffen, P. E. J. Van Der Meijden, and J. W. M. Heemskerk. Localized endothelial-based control of platelet aggregation and coagulation under flow: a proof-of-principle vessel-on-achip study. J. Thromb. Haemost. 18:931-941, 2020.

${ }^{3}$ Burrell, L. M., C. I. Johnston, C. Tikellis, and M. E. Cooper. ACE2, a new regulator of the renin-angiotensin system. Trends Endocrinol. Metab. 15:166-169, 2004.

${ }^{4}$ Chang, S. Y., et al. Human liver-kidney model elucidates the mechanisms of aristolochic acid nephrotoxicity. JCI Insight 2:e95978, 2017.

${ }^{5}$ Crane, J. T., and J. F. Riley. Design of BSL3 laboratories (chapter 7). J. Am. Biol. Saf. Assoc. 4:17-23, 1999.

${ }^{6}$ Davidson, M. D., J. A. Burdick, and R. G. Wells. Engineered biomaterial platforms to study fibrosis. $A d v$. Healthc. Mater. 9:1901682, 2020. https://doi.org/10.1002/ adhm.201901682.

${ }^{7}$ Dehne, E.-M., and U. Marx. Human body-on-a-chip systems. In: Organ-On-a-Chip. Amsterdam: Elsevier, 2020, pp. 429-439.

${ }^{8}$ Denker, B. M., and E. Sabath. The biology of epithelial cell tight junctions in the kidney. J. Am. Soc. Nephrol. 22:622625, 2011.
${ }^{9}$ Diao, B., et al. Human kidney is a target for novel severe acute respiratory syndrome coronavirus 2 (SARS-CoV-2) infection. medRxiv 2020. https://doi.org/10.1101/2020.03.0 4.20031120 .

${ }^{10}$ Enjuanes, L., S. Zuñiga, C. Castaño-Rodriguez, J. Gutierrez-Alvarez, J. Canton, and I. Sola. Molecular basis of coronavirus virulence and vaccine development. In: Advances in Virus Research. Amsterdam: Academic Press Inc., 2016, pp. 245-286.

${ }^{11}$ Frieman, M., et al. Molecular determinants of severe acute respiratory syndrome coronavirus pathogenesis and virulence in young and aged mouse models of human disease. $J$. Virol. Am. Soc. Microbiol. 86:884-897, 2012.

${ }^{12}$ Frohlich, E. M., J. L. Alonso, J. T. Borenstein, X. Zhang, M. A. Arnaout, and J. L. Charest. Topographically-patterned porous membranes in a microfluidic device as an in vitro model of renal reabsorptive barriers. Lab Chip 13:2311-2319, 2013.

${ }^{13}$ Gao, X., Y. Tanaka, Y. Sugii, K. Mawatari, and T. Kitamori. Basic structure and cell culture condition of a bioartificial renal tubule on chip towards a cell-based separation microdevice. Anal. Sci. 27:907-912, 2011.

${ }^{14}$ Geuens, T., C. A. van Blitterswijk, and V. L. S. LaPointe. Overcoming kidney organoid challenges for regenerative medicine. NPJ Regen. Med. 5:8, 2020.

${ }^{15}$ Gopalakrishnan, A., A. Mossaid, K. B. Lo, V. Vasudevan, P. A. McCullough, and J. Rangaswami. Fulminant acute kidney injury in a young patient with novel coronavirus 2019. Cardiorenal Med. 1-6, 2020.

${ }^{16}$ Hassan, S., et al. Liver-on-a-chip models of fatty liver disease. Hepatology 71:733-740, 2020. https://doi.org/10.1 002/hep.31106.

${ }^{17}$ Homan, K. A., et al. Bioprinting of 3D convoluted renal proximal tubules on perfusable chips. Sci. Rep. 6:34845, 2016.

${ }^{18}$ Hong, K. S., et al. Clinical features and outcomes of 98 patients hospitalized with SARS-CoV-2 infection in Daegu, South Korea: a brief descriptive study. Yonsei Med. J. 61:431-437, 2020. https://doi.org/10.3349/ymj.2020.61.5.4 31.

${ }^{19}$ Interim Guidelines for Biosafety and COVID-19 | CDC. Centers Dis. Control Prev., 2020. https://www.cdc.gov/cor onavirus/2019-ncov/lab/lab-biosafety-guidelines.html.

${ }^{20}$ Jang, K. J., H. S. Cho, D. H. Kang, W. G. Bae, T. H. Kwon, and K. Y. Suh. Fluid-shear-stress-induced translocation of aquaporin-2 and reorganization of actin cytoskeleton in renal tubular epithelial cells. Integr. Biol. 3:134-141, 2011.

${ }^{21}$ Jang, K. J., and K. Y. Suh. A multi-layer microfluidic device for efficient culture and analysis of renal tubular cells. Lab Chip 10:36-42, 2010.

${ }^{22}$ Jang, K. J., et al. Human kidney proximal tubule-on-a-chip for drug transport and nephrotoxicity assessment. Integr. Biol. 5:1119-1129, 2013.

${ }^{23}$ Jansen, J., et al. Human proximal tubule epithelial cells cultured on hollow fibers: living membranes that actively transport organic cations. Sci. Rep. 5:1-12, 2015.

${ }^{24}$ Kim, S., H. Lee, M. Chung, and N. L. Jeon. Engineering of functional, perfusable 3D microvascular networks on a chip. Lab Chip 13:1489-1500, 2013.

${ }^{25}$ Kim, S., S. C. LesherPerez, B. C. C. Kim, C. Yamanishi, J. M. Labuz, B. Leung, and S. Takayama. Pharmacokinetic profile that reduces nephrotoxicity of gentamicin in a perfused kidney-on-a-chip. Biofabrication 8:015021, 2016. 
${ }^{26} \mathrm{Kim}$, S. J., et al. Hydrogels with an embossed surface: an all-in-one platform for mass production and culture of human adipose-derived stem cell spheroids. Biomaterials 188:198-212, 2019.

${ }^{27}$ Kimura, H., Y. Sakai, and T. Fujii. Organ/body-on-a-chip based on microfluidic technology for drug discovery. Drug Metab. Pharmacokinet. 33:43-48, 2018.

${ }^{28} \mathrm{Li}, \mathrm{Z}$., et al. Assessment of cadmium-induced nephrotoxicity using a kidney-on-a-chip device. Toxicol. Res. 6:372380, 2017.

${ }^{29} \mathrm{Li}, \mathrm{Z}$., et al. Assessment of hepatic metabolism-dependent nephrotoxicity on an organs-on-a-chip microdevice. Toxicol. Vitro 46:1-8, 2018.

${ }^{30} \mathrm{Li}, \mathrm{Z}$., et al. Caution on kidney dysfunctions of 2019-nCoV patients. medRxiv 2020. https://doi.org/10.1101/2020.02.08. 20021212.

${ }^{31}$ Lipphardt, M., et al. Dickkopf-3 in aberrant endothelial secretome triggers renal fibroblast activation and endothelial-mesenchymal transition. Nephrol. Dial Transpl. 34:49-62, 2019.

${ }^{32}$ Maschmeyer, I., et al. A four-organ-chip for interconnected long-term co-culture of human intestine, liver, skin and kidney equivalents. Lab Chip 15:2688-2699, 2015.

${ }^{33}$ Pacciarini, F., et al. Persistent replication of severe acute respiratory syndrome coronavirus in human tubular kidney cells selects for adaptive mutations in the membrane protein. J. Virol. 82:5137-5144, 2008.

${ }^{34}$ Pan, X.-W., D. Xu, H. Zhang, W. Zhou, L.-H. Wang, and X.-G. Cui. Identification of a potential mechanism of acute kidney injury during the COVID-19 outbreak: a study based on single-cell transcriptome analysis. Intensive Care Med. 2020. https://doi.org/10.1007/s00134-020-06026-1.

${ }^{35}$ Petrosyan, A., et al. A glomerulus-on-a-chip to recapitulate the human glomerular filtration barrier. Nat. Commun. 10:1-17, 2019.

${ }^{36} \mathrm{Qu}$, Y., et al. A nephron model for study of drug-induced acute kidney injury and assessment of drug-induced nephrotoxicity. Biomaterials 155:41-53, 2018.

${ }^{37}$ Raghavan, V., Y. Rbaibi, N. M. Pastor-Soler, M. D. Carattino, and O. A. Weisz. Shear stress-dependent regulation of apical endocytosis in renal proximal tubule cells mediated by primary cilia. Proc. Natl. Acad. Sci. USA 111:8506-8511, 2014.

${ }^{38}$ Salminen, A. T., et al. Ultrathin dual-scale nano- and microporous membranes for vascular transmigration models. Small 15:e1804111, 2019.

${ }^{39}$ Shanti, A., J. Teo, and C. Stefanini. In vitro immune organs-on-chip for drug development: a review. Pharmaceutic 10:278, 2018.

${ }^{40}$ Shi, J., et al. Susceptibility of ferrets, cats, dogs, and other domesticated animals to SARS-coronavirus 2. Science 368:1016-1020, 2020.

${ }^{41}$ Simon-Friedt, B. R., M. J. Wilson, D. A. Blake, H. Yu, Y. Eriksson, and J. K. Wickliffe. The RPTEC/TERT1 cell line as an improved tool for in vitro nephrotoxicity assessments. Biol. Trace Elem. Res. 166:66-71, 2015.

${ }^{42}$ Singh, N. K., et al. Three-dimensional cell-printing of advanced renal tubular tissue analogue. Biomaterials 232:119734, 2020.

${ }^{43}$ Snouber, L. C., et al. Analysis of transcriptomic and proteomic profiles demonstrates improved Madin-Darby canine kidney cell function in a renal microfluidic biochip. Biotechnol. Prog. 28:474-484, 2012.
${ }^{44}$ Suter-Dick, L., et al. Combining extracellular miRNA determination with microfluidic 3D cell cultures for the assessment of nephrotoxicity: a proof of concept study. AAPS J. 20:86, 2018

${ }^{45}$ Teoh, K. T., et al. The SARS coronavirus E protein interacts with PALS1 and alters tight junction formation and epithelial morphogenesis. Mol. Biol. Cell 21:38383852, 2010.

${ }^{46}$ Theobald, J., et al. In vitro metabolic activation of vitamin D3 by using a multi-compartment microfluidic liver-kidney organ on chip platform. Sci. Rep. 9:1-11, 2019.

${ }^{47}$ Tian, H., et al. A novel tissue-based liver-kidney-on-a-chip can mimic liver tropism of extracellular vesicles derived from breast cancer cells. Biotechnol. J. 15:1900107, 2020. h ttps://doi.org/10.1002/biot.201900107.

${ }^{48}$ Verschuren, E. H. J., C. Castenmiller, D. J. M. Peters, F. J. Arjona, R. J. M. Bindels, and J. G. J. Hoenderop. Sensing of tubular flow and renal electrolyte transport. Nat. Rev. Nephrol. 16:337-351, 2020.

${ }^{49}$ Vormann, M. K., et al. Nephrotoxicity and kidney transport assessment on 3D perfused proximal tubules. AAPS J. 20:90, 2018

${ }^{50}$ Vriend, J., et al. Flow stimulates drug transport in a human kidney proximal tubule-on-a-chip independent of primary cilia. Biochim. Biophys. Acta Gen. Subj. 1864:129433, 2020.

${ }^{51}$ Wang, L., T. Tao, W. Su, H. Yu, Y. Yu, and J. Qin. A disease model of diabetic nephropathy in a glomerulus-ona-chip microdevice. Lab Chip 17:1749-1760, 2017.

${ }^{52}$ Wang, J., C. Wang, N. Xu, Z.-F. Liu, D.-W. Pang, and Z.L. Zhang. A virus-induced kidney disease model based on organ-on-a-chip: pathogenesis exploration of virus-related renal dysfunctions. Biomaterials 2019. https://doi.org/10.1 016/j.biomaterials.2019.119367.

${ }^{53}$ Wang, L., et al. Coronavirus disease 19 infection does not result in acute kidney injury: an analysis of 116 hospitalized patients from Wuhan, China. Am. J. Nephrol. 51:343-348, 2020.

${ }^{54} \mathrm{Xia}, \mathrm{X}$., et al. Analyzing the early CT findings and clinical features of 12 patients with 2019 novel coronavirus disease (COVID-19) in China. Iran. Red Crescent Med. J. 22:e103460, 2020

${ }^{55}$ Xiaohong, Y., et al. A pathological report of three COVID19 cases by minimal invasive autopsies. Chin. J. Pathol. 49:E009, 2020.

${ }^{56} \mathrm{Xu}, \mathrm{Y} .-\mathrm{H}$., et al. Clinical and computed tomographic imaging features of novel coronavirus pneumonia caused by SARS-CoV-2. J. Infect. 80:394-400, 2020. https://doi. org/10.1016/j.jinf.2020.02.017.

${ }^{57}$ Ye, Z., Y. Zhang, Y. Wang, Z. Huang, and B. Song. Chest CT manifestations of new coronavirus disease 2019 (COVID-19): a pictorial review. Eur. Radiol. 2020. https://d oi.org/10.1007/s00330-020-06801-0.

${ }^{58}$ Zhou, M., H. Ma, H. Lin, and J. Qin. Induction of epithelial-to-mesenchymal transition in proximal tubular epithelial cells on microfluidic devices. Biomaterials 35:1390-1401, 2014.

${ }^{59}$ Zihni, C., M. S. Balda, and K. Matter. Signalling at tight junctions during epithelial differentiation and microbial pathogenesis. J. Cell Sci. 127:3401-3413, 2014.

Publisher's Note Springer Nature remains neutral with regard to jurisdictional claims in published maps and institutional affiliations. 